\title{
ON THE EXPECTED NUMBER OF ASSIGNMENTS IN REDUCED MATRICES FOR THE LINEAR ASSIGNMENT PROBLEM
}

\author{
W.M. NAWIJN and B. DORHOUT \\ Faculty of Applied Mathematics, University of Twente, P.O. Box 217, 7500 AE Enschede, The Netherlands \\ Received September 1988 \\ Revised April 1989
}

\begin{abstract}
A linear $n \times n$ assignment problem is considered for which the elements of the cost matrix are sampled from a continuous probability distribution. Based on the zero entries of the reduced matrix the expectation of the maximum number of initial assignments is determined for general $n$, as well as an asymptotic value for large $n$.
\end{abstract}

linear assignment problem * matching* bipartite graph*occupancy problem

\section{Introduction}

Efficient algorithms for solving the linear assignment problem often start by reducing the cost matrix $\left(c_{i j}\right), 1 \leqslant i, j \leqslant n$, see e.g. $[1,5]$.

The reduced matrix is then used for constructing an initial set of assignments, where an assignment coincides with one of the zero elements in the reduced matrix. We consider stochastic assignment problems in which the cost coefficients $c_{i j}$ are random variables satisfying the following two assumptions:

1. $\left\{c_{i j}, 1 \leqslant i, j \leqslant n\right\}$ constitute a family of independent identically distributed random variables with distribution function $F$;

2. $F$ is continuous.

In view of the latter assumption, when reducing a row or column of the matrix, one obtains exactly one zero element with probability one.

Our aim is to derive, within this class of assignment problems, an expression for the expected number of assignments based on the reduced cost matrix obtained by a particular start procedure. This procedure consists of a reduction procedure and a subsequent assignment procedure.

The reduction procedure consists of two phases: a row reduction and a column reduction, and proceeds in the following way.

Phase I (row reduction). Let $J$ be the set of all columns and $R$ be the set of all rows. For each row $i \in R$ determine $j_{i}$ such that $c_{i j_{i}}=\min _{1 \leqslant j \leqslant n} c_{i j}$, which is unique with probability one.

Reduce each row in the matrix by subtracting its minimum element and let $c_{i j}^{*}=c_{i j}-c_{i j,}, i \in \mathbb{R}, j \in J$.

Denote by

$$
J_{\mathrm{p}}=\left\{j \in J \mid \text { for some } i \in R: c_{i j}^{*}=0\right\}
$$

the set of columns containing at least one zero element, and let $J_{\mathrm{e}}=J \backslash J_{\mathrm{p}}$.

Phase II (column reduction of the set $J_{\mathrm{e}}$ ). For each column $j \in J_{\mathrm{e}}$ determine $i_{j}$ such that $c_{i_{j}, j}^{*}=$ $\min _{1 \leqslant i \leqslant n} c_{i j}^{*}$ which is unique with probability one. 
Reduce each column $j \in J_{\mathrm{e}}$ by subtracting the column's minimum:

$$
\tilde{c}_{i j}=c_{i j}^{*}-c_{i_{j}, j}^{*}, \quad i \in R, \quad j \in J_{\mathrm{e}} .
$$

Denote by

$$
R_{\mathrm{p}}=\left\{i \in R \mid \text { for some } j \in J_{\mathrm{e}}: \tilde{c}_{i j}=0\right\}
$$

the set of rows containing at least one zero element obtained by column reduction.

Now the assignment procedure runs as follows:

Step 1: Assign to each row $i \in R_{\mathrm{p}}$ one of the columns in $J_{\mathrm{e}}$;

Step 2: Elimination step. Eliminate from $J_{\mathrm{p}}$ the subset $S$ of columns whose zero elements are fully contained in $R_{\mathrm{p}}$, so $S=\left\{j \in J_{\mathrm{p}} \mid\right.$ all $\left.i_{j} \in R_{\mathrm{p}}\right\}$. Let $J_{\mathrm{p}}{ }^{*}=J_{\mathrm{p}} \backslash S$.

Step 3: Assign to each column $j \in J_{\mathrm{p}}{ }^{*}$ one of the rows in $R$ (i.c. $R \backslash R_{\mathrm{p}}$ ), which is always possible.

It should be noticed that the procedure is unambiguous only for random instances with unique minima in rows and columns. This however is the case with probability one by virtue of Assumption 2 .

Observe that the total number of assignments, $V$ say, equals

$$
\begin{aligned}
V & =\left|R_{\mathrm{p}}\right|+\left|J_{\mathrm{p}}{ }^{*}\right|=\left|R_{\mathrm{p}}\right|+\left|J_{\mathrm{p}}\right|-|S| \cdot \\
& =\left|R_{\mathrm{p}}\right|+n-\left|J_{\mathrm{e}}\right|-|S| .
\end{aligned}
$$

The reduction procedure generates a (random) bipartite graph $G=(R, J, E)$ with $(i, j) \in E, i \in R$, $j \in J$, if $c_{i j}^{*}=0$ or $\tilde{c}_{i j}=0$. The assignment procedure described above generates a matching $M$ on $G$ with $|M|=V$. From this procedure it is not difficult to see that $R_{\mathrm{p}} \cup J_{\mathrm{p}}^{*}$ is a covering of $G$. Since $V=|M|=\left|R_{\mathrm{p}}\right|+\left|J_{\mathrm{p}}^{*}\right|, M$ is a maximum matching by the König-Egervary theorem.

In Section 1 we derive, for general $n$, the expected value EV over all random instances, while in Section 2 we will consider the limiting value of $\mathrm{EV} / n$ as $n$ tends to infinity.

\section{The solution for general $n$}

Let us introduce the following random variables: $X=\left|J_{\mathrm{e}}\right|$, which denotes the number of columns not containing zero elements after row reduction; $Z_{j}$, the number of zero elements in column $j$ after row reduction; $Y=\left|R_{\mathrm{p}}\right|$, denoting the number of different rows getting at least one zero element by column reduction (only in case $X>0$ ), and finally, the binary variable $W_{j}$ which equals one if column $j$ is eliminated in the assignment procedure (i.e. if $j \in S$ ), and equals zero otherwise. Note that $\sum_{j=1}^{n} W_{j}=|S|$. The expectation of $V$ is formally given by, cf. (1),

$$
\mathrm{EV}=\mathrm{EY}+n-\mathrm{EX}-\sum_{j=1}^{n} \mathrm{EW}_{j}
$$

By symmetry $\mathrm{EW}_{1}=\mathrm{EW}_{2}=\cdots=\mathrm{EW}_{n}=\mathrm{EW}$, therefore

$$
\mathrm{EV}=\mathrm{EY}+n-\mathrm{EX}-n \mathrm{EW}
$$

in which EW is the probability that an arbitrary column will belong to the set $S$.

The determination of EV is based upon the following two observations.

Observation 1. Since all $c_{i j}$ are i.i.d. random variables, the column numbers $j_{i}(i=1,2, \ldots, n)$ satisfy $P\left(j_{i}=k\right)=1 / n(1 \leqslant k \leqslant n)$ irrespective of $F$. In other words the row reduction can be interpreted as randomly allocating $n$ balls (i.e. the rows) into $n$ cells (i.e. the columns). The latter model is known as an occupancy model, see e.g. Feller [2], and Johnson and Kotz [3]. It should be noted that the present as well as the next observation essentially rests on the assumptions regarding the random variables $c_{i j}$. 
Observation 2. Suppose that the row reduction leaves $k$ columns empty, i.e. $X=k$, and let $J_{\mathrm{e}}=$ $\left\{r_{1}, r_{2}, \ldots, r_{k}\right\}$ denote these columns. The independent random variables $c_{i r_{j}}^{*}(1 \leqslant i \leqslant n, 1 \leqslant j \leqslant k)$ still have the same (conditional) probability distribution, since all the row minima $c_{i j_{i}}$ are identically distributed. Hence, the column reduction can likewise be interpreted in terms of the classical occupancy model.

All terms on the right in (3) can be found using known results for the occupancy problem. The first result we need is given in the next lemma, see Johnson and Kotz [3, p.144] and Feller [2, p.92].

Lemma 1. Let $X_{n}(r)$ be the number of empty cells after randomly allocating $r$ balls into $n$ cells. Then

(a)

$$
\operatorname{EX}_{n}(r)=n\left(1-\frac{1}{n}\right)^{r}
$$

(b)

$$
\operatorname{var} X_{n}(r)=n(n-1)\left(1-\frac{2}{n}\right)^{r}+n\left(1-\frac{1}{n}\right)^{r}-n^{2}\left(1-\frac{1}{n}\right)^{2 r}
$$

(c)

$$
\begin{aligned}
p_{k}(r, n) & \stackrel{\mathrm{d}}{=} P\left(X_{n}(r)=k\right)=\left(\begin{array}{l}
n \\
k
\end{array}\right)\left(1-\frac{k}{n}\right)^{r} p_{0}(r, n-k) \\
& =\left(\begin{array}{l}
n \\
k
\end{array}\right)\left(1-\frac{k}{n}\right)^{r} \sum_{j=0}^{n-k}(-1)^{j}\left(\begin{array}{c}
n-k \\
j
\end{array}\right)\left(1-\frac{j}{n-k}\right)^{r} \\
& =\left(\begin{array}{l}
n \\
k
\end{array}\right) \sum_{j=k}^{n}(-1)^{j+k}\left(\begin{array}{c}
n-k \\
j-k
\end{array}\right)\left(1-\frac{j}{n}\right)^{r} \quad \text { for } \max (0, n-r) \leqslant k \leqslant n, r \geqslant 1 ; \\
p_{n}(r, n) & =0, \quad r \geqslant 1 \quad \text { and } \quad p_{n}(0, n)=1 .
\end{aligned}
$$

From part (a) of the lemma we immediately have

$$
\mathrm{EX}=\mathrm{EX}_{n}(n)=n\left(1-\frac{1}{n}\right)^{n}
$$

From Observation 2 and the above lemma it follows that

$$
E(Y \mid X=k)=n-n\left(1-\frac{1}{n}\right)^{k}, \quad k=0,1, \ldots, n-1 .
$$

Hence

$$
\mathrm{EY}=n-n E\left\{\left(1-\frac{1}{n}\right)^{x}\right\}
$$

Using part (c) of the lemma, this can be written as

$$
\mathrm{EY}=n-n \sum_{k=0}^{n}\left(1-\frac{1}{n}\right)^{k}\left(\begin{array}{l}
n \\
k
\end{array}\right) \sum_{j=k}^{n}(-1)^{j+k}\left(\begin{array}{c}
n-k \\
j-k
\end{array}\right)\left(1-\frac{j}{n}\right)^{n}
$$

in which the term $k=n$ has contribution zero. Interchanging the order of summation, rewriting the binomial coefficients and using the binomial formula this expression can be simplified to

$$
\mathrm{EY}=n-n \sum_{j=0}^{n}(-1)^{j}\left(\begin{array}{l}
n \\
j
\end{array}\right)\left(1-\frac{j}{n}\right)^{n} n^{-j}
$$


Let us now turn to the determination of $\mathrm{EW}$, in particular we consider the probability that some arbitrarily chosen column will be contained in $J_{\mathrm{p}}$ and will subsequently be eliminated, so that it will ultimately belong to $S$. This probability can be expressed as

$$
\mathrm{EW}=\sum_{k=1}^{n-1} \sum_{j=1}^{k+1} P(W=1 \mid Z=j, X=k) P(Z=j, X=k)
$$

noting that $P(W=1 \mid Z=k+1, X=k)=0$.

The probability $P(Z=j, X=k)$ is equivalent to the probability that a random allocation of $n$ balls to $n$ cells leaves $k$ cells empty and such that a particular cell will be occupied by exactly $j$ balls.

Hence, cf. Lemma 1, part (c),

$$
P(Z=j, X=k)=\left(\begin{array}{l}
n \\
j
\end{array}\right) \frac{1}{n^{j}}\left(1-\frac{1}{n}\right)^{n-j} p_{k}(n-j, n-1) .
$$

The probability $P(W=1 \mid Z=j, X=k)$ can be interpreted in terms of the classical occupancy problem as the probability that each of $j$ given cells (i.e. the rows containing the zero elements in an arbitrary column) is occupied, after randomly allocating $k$ balls into $n$ cells. The corresponding probability is given by, see Feller [1, p.59],

$$
P(W=1 \mid Z=j, X=k)=\sum_{i=0}^{j}(-1)^{i}\left(\begin{array}{l}
j \\
i
\end{array}\right)\left(1-\frac{i}{n}\right)^{k} .
$$

Note that the expression on the right equals zero for $j>k$.

From (10) and (11) we obtain

$$
\mathrm{EW}=\sum_{k=1}^{n-1} \sum_{j=1}^{k+1} \sum_{i=0}^{j}(-1)^{i}\left(\begin{array}{l}
j \\
i
\end{array}\right)\left(1-\frac{i}{n}\right)^{k}\left(\begin{array}{l}
n \\
j
\end{array}\right) \frac{1}{n^{j}}\left(1-\frac{1}{n}\right)^{n-j} p_{k}(n-j, n-1) .
$$

Introducing the indicator function $I(\cdot)$ this can be written more principally as

$$
\mathrm{EW}=E\left[\left\{\sum_{i=0}^{Z}(-1)^{i}\left(\begin{array}{c}
Z \\
i
\end{array}\right)\left(1-\frac{i}{n}\right)^{X}\right\} I(Z \geqslant 1, X \geqslant 1)\right]
$$

The value of the expression does not change when replacing $I(Z \geqslant 1, X \geqslant 1)$ by $I(Z \geqslant 1, X \geqslant 0)=I(Z \geqslant 1)$, so

$$
\mathrm{EW}=E\left[\left\{\sum_{i=0}^{Z}(-1)^{k}\left(\begin{array}{c}
Z \\
i
\end{array}\right)\left(1-\frac{i}{n}\right)^{x}\right\} I(Z \geqslant 1)\right]
$$

a result which will be used in the sequel.

Summarizing, the general solution for EV can be obtained from (3), (4), (8) and (12).

\section{An asymptotic result for EV $(n \rightarrow \infty)$}

To derive a limiting expression for $\mathrm{EV} / n$ as $n \rightarrow \infty$, we will need the results given in the next three lemmas. First observe from Lemma 1 that the fraction of empty cells tends to 1 /e with probability one when $n \rightarrow \infty$ and $r / n \rightarrow 1$ by Chebyshev's inequality.

Lemma 2. Let $X_{n}(r)$ be the number of empty cells after randomly allocating $r$ balls into $n$ cells. If $r \rightarrow \infty$ and $n \rightarrow \infty$ such that $r / n \rightarrow 1$, then

$$
\lim _{n \rightarrow \infty} \frac{1}{n} X_{n}(r)=\frac{1}{\mathrm{e}} \text { with probability one. }
$$

We also need the simultaneous limit distribution of $X$ and $Z$, given in the following lemma. 
Lemma 3. For $j=0,1, \ldots$, and $x \in \mathbb{R}$,

$$
\lim _{n \rightarrow \infty} P\left(Z=j, \frac{1}{n} X_{n}(n) \leqslant x\right)=\frac{\mathrm{e}^{-1}}{j !} U\left(x-\frac{1}{\mathrm{e}}\right),
$$

where $U(x)=1$ for $x \geqslant 0$ and $U(x)=0$ for $x<0$.

Proof. Since

$$
\begin{aligned}
P\left(Z=j, \frac{1}{n} X_{n}(n) \leqslant x\right) & =P\left(\frac{1}{n} X_{n}(n) \leqslant x \mid Z=j\right) P(Z=j) \\
& =P\left(\frac{1}{n} X_{n-1}(n-j) \leqslant x\right) P(Z=j)
\end{aligned}
$$

and, moreover,

$$
P(Z=j)=\left(\begin{array}{c}
n \\
j
\end{array}\right) n^{-j}\left(1-\frac{1}{n}\right)^{n-j}, \quad 0 \leqslant j \leqslant n,
$$

relation (14) is readily verified by applying Lemma 2 and noting that the binomial distribution (15) tends to a Poisson distribution with mean 1.

Lemma 4. Let the random variable $A_{n}$ be such that $0 \leqslant A_{n} \leqslant n$ and $A_{n} / n \stackrel{a . s}{\rightarrow} c ;$ then

$$
\lim _{n \rightarrow \infty} E\left\{\left(1-\frac{1}{n}\right)^{A_{n}}\right\}=\exp (-c)
$$

Proof. Applying the inequality $\mathrm{e}^{-t /(1-t)} \leqslant 1-t \leqslant \mathrm{e}^{-t}, 0<t<1$, it follows that

$$
E\left\{\exp \left(-\frac{A_{n}}{n-1}\right)\right\} \leqslant E\left(1-\frac{1}{n}\right)^{A_{n}} \leqslant E\left\{\exp \left(-\frac{A_{n}}{n}\right)\right\}
$$

Letting $n \rightarrow \infty$ the assertion follows from Helly-Bray's theorem, see Loève [4, p.182].

Starting from (3) we have

$$
\lim _{n \rightarrow \infty} \frac{1}{n} \mathrm{EV}=1+\lim _{n \rightarrow \infty} \frac{1}{n} \mathrm{EY}-\lim _{n \rightarrow \infty} \frac{1}{n} \mathrm{EX}-\lim _{n \rightarrow \infty} \mathrm{EW},
$$

provided the limits exist. From (4) we have

$$
\lim _{n \rightarrow \infty} \frac{1}{n} \mathrm{EX}=\frac{1}{\mathrm{e}} \text {. }
$$

From (6) it follows that

$$
\lim _{n \rightarrow \infty} \frac{1}{n} E Y=1-\lim _{n \rightarrow \infty} E\left\{\left(1-\frac{1}{n}\right)^{x}\right\} .
$$

Hence, by (17) and Lemma 4, we have

$$
\lim _{n \rightarrow \infty} \frac{1}{n} \mathrm{EY}=1-\lim _{n \rightarrow \infty} \frac{1}{n} \mathrm{EX}_{n}\left(X_{n}(n)\right)=1-\exp (-1 / \mathrm{e}) .
$$

Finally we consider the limit, cf. (13),

$$
\lim _{n \rightarrow \infty} \mathrm{EW}=\lim _{n \rightarrow \infty} E\left[\left\{\sum_{i=0}^{Z}(-1)^{i}\left(\begin{array}{c}
Z \\
i
\end{array}\right)\left(1-\frac{i}{n}\right)^{X}\right\} I(Z \geqslant 1)\right] .
$$


We will show that this limit equals

$$
\lim _{n \rightarrow \infty} \mathrm{EW}=\lim _{n \rightarrow \infty} E\left[\{1-\exp (-X / n)\}^{Z} I(Z \geqslant 1)\right]
$$

from which we obtain the final result.

To prove the equivalence of $(20)$ and (21), consider the absolute difference

$$
\begin{aligned}
A & =\left|E\left[\sum_{i=0}^{Z}(-1)^{i}\left(\begin{array}{c}
Z \\
i
\end{array}\right)\left\{\left(1-\frac{i}{n}\right)^{X}-\mathrm{e}^{-i X / n}\right\} I(Z \geqslant 1)\right]\right| \\
& \leqslant E\left[\sum_{i=0}^{Z}\left(\begin{array}{c}
Z \\
i
\end{array}\right)\left|\mathrm{e}^{-i X / n}-\left(1-\frac{i}{n}\right)^{X}\right| I(Z \geqslant 1)\right] .
\end{aligned}
$$

Since for $0 \leqslant i \leqslant n$ and $X \geqslant 1$

$$
\begin{aligned}
\mathrm{e}^{-i X / n}-\left(1-\frac{i}{n}\right)^{X} & =\left\{\mathrm{e}^{-i / n}-1+\frac{i}{n}\right\} \sum_{j=0}^{X-1} \mathrm{e}^{-j i / n}\left(1-\frac{i}{n}\right)^{X-j-1} \\
& \leqslant\left\{\mathrm{e}^{-i / n}-1+\frac{i}{n}\right\} X \leqslant i^{2} X /\left(2 n^{2}\right)
\end{aligned}
$$

we obtain

$$
A \leqslant E\left[\sum_{i=0}^{Z}\left(\begin{array}{c}
Z \\
i
\end{array}\right) \frac{i^{2} X}{2 n^{2}} I(Z \geqslant 1)\right] \leqslant \frac{1}{2 n} E\left[\sum_{i=0}^{Z} i^{2}\left(\begin{array}{c}
Z \\
i
\end{array}\right)\right]
$$

since $X<n$.

From (15) it is readily verified that the right hand side of this relation is equal to

$$
\frac{1}{2 n}\left\{\left(1-\frac{1}{n}\right)\left(1+\frac{1}{n}\right)^{n-2}+\left(1+\frac{1}{n}\right)^{n-1}\right\},
$$

therefore $A=\mathrm{O}\left(n^{-1}\right)$ for $n \rightarrow \infty$, which proves (21).

Applying Lemma 3 and Helly-Bray's theorem to (21) we conclude that

$$
\begin{aligned}
\lim _{n \rightarrow \infty} \mathrm{EW} & =\frac{1}{\mathrm{e}} \sum_{j=1}^{\infty} \frac{1}{j !}(1-\exp (-1 / \mathrm{e}))^{j} \\
& =\exp (-\exp (-1 / \mathrm{e}))-1 / \mathrm{e}
\end{aligned}
$$

Summarizing, we have proved the following theorem, see (16), (17), (19) and (22):

Theorem. The expected maximum number of assignments in the reduced matrix (or expected cardinality of a maximum matching in $G$ ) satisfies

$$
\begin{aligned}
\lim _{n \rightarrow \infty} \frac{1}{n} \mathrm{EV} & =2-\exp (-1 / \mathrm{e})-\exp (-\exp (-1 / \mathrm{e})) \\
& \cong 0.8073
\end{aligned}
$$

Recalling the definition of $X_{n}(r)$, cf. Lemma 1, observe from Lemmas 1 and 4 that the above limit can be represented probabilistically as

$$
\begin{aligned}
\lim _{n \rightarrow \infty} \frac{1}{n} \mathrm{EV}_{2} & =\lim _{n \rightarrow \infty} \frac{1}{n} E\left\{2 n-X_{n}\left(X_{n}(n)\right)-X_{n}\left(X_{n}\left(X_{n}(n)\right)\right)\right\} \\
& =\lim _{n \rightarrow \infty} \frac{1}{n} E\left\{Y+n-X_{n}(n-Y)\right\} .
\end{aligned}
$$

It should be noted, however, that for finite $n, \mathrm{EX}_{n}(n-Y) \neq \mathrm{EX}+n \mathrm{EW}$, cf. (16). 
Table 1

Exact results

\begin{tabular}{llllllllll}
\hline$n$ & 2 & 3 & 4 & 5 & 10 & 15 & 20 & 30 & 40 \\
\hline $\mathrm{EV} / n$ & 1.000 & 0.914 & 0.877 & 0.860 & 0.831 & 0.823 & 0.819 & 0.815 & 0.813 \\
\hline
\end{tabular}

From Lemma $1(\mathrm{~b})$ it is readily verified that $\operatorname{Var}\left(V_{2} / n\right) \rightarrow 0$ as $n \rightarrow \infty$, so one may expect this to hold for $V / n$ as well, a fact supported by simulation.

In Table 1 we give some exact results for $\mathrm{EV} / n$ which show the decrease towards the asymptotic value.

\section{References}

[1] B. Dorhout, "The shortest augmenting path method for solving the linear assignment problem", Memorandum 453, Department of Applied Mathematics, University of Twente, 1984.

[2] W. Feller, An Introduction to Probability Theory and its Applications Vol. I, 2nd ed., Wiley, New York, 1964.

[3] N.L. Johnson and S. Kotz, Urn Models and their Application, Wiley, New York, 1977.

[4] M. Loève, Probability Theory, 3rd ed., Van Nostrand, Princeton, 1963.

[5] D.T. Phillips, A. Ravindran and J.J. Solberg, Operations Research, Wiley, New York, 1976. 